\title{
Barriers to shared care for pre-school oral health
}

\author{
A qualitative study exploring barriers to a model of shared care for pre-school children's oral health \\ M. G. Gussy, E. Waters and N. M. Kilpatrick Br Dent J 2006; 200: 165-170
}

\section{Objective}

To explore the oral health beliefs and practices of primary health care professionals which may act as barriers to the development of a model of shared care for the oral health of pre-school children. Design

Qualitative focus group discussions and semi-structured interviews. Setting

Four rural local government areas in Victoria, Australia, 2003.

Subjects and methods

Subjects: maternal and child health nurses, general medical practitioners, dental professionals and paediatricians working in the four local government areas. Data collection: discipline specific focus groups and semi-structured interviews. Data analysis: transcription, coding, clustering and thematic analysis.

Results

Several strong themes emerged from the data. All participants agreed that dental caries is a significant health issue for young children and their families. Beliefs about the aetiology of dental caries and its prevention were variable and often simplistic, focusing predominantly on diet. Dental professionals did not believe that they had a primary role in the oral health of pre-school aged children but that others particularly maternal and child health nurses did. However other health care professionals were not confident in assuming this role.

Conclusions

This study has identified important barriers and possible strategies for the development of an integrated and shared approach to preventing dental caries in pre-school aged children. Clear and consistent oral health information and agreed roles and responsibilities need to be developed.

\section{IN BRIEF}

- Non-dental healthcare professionals consider dental decay to be a significant problem for pre-school aged children.

- Conflicting information provided by healthcare professionals creates confusion and discourages parents from accessing oral healthcare services.

- Differences in perception between GPs, dentists and community nurses in their roles and responsibilities are a significant barrier to improving the oral health of pre-school aged children.

- Despite strong evidence to support anticipatory guidance in general, the dental profession has yet to fully embrace this concept.

\section{COMMENT}

This paper's objective was to investigate the oral health beliefs and practices of primary health care professionals, which may act as barriers to the development of a model of shared care for oral health of pre-school children in Australia. In Australia there are significant inequalities in pre-school dental caries in socially deprived areas. Twelve percent of two-year old Australians have never seen a dental professional. One of the main challenges is that there is little communication between any of the health care professionals. Therefore, this study looked at the feasibility and effectiveness of a model of shared care applied to dental caries in relation to young children in disadvantaged communities.

The authors used a qualitative methodology to identify the participants' perceptions of important issues associated with the oral health of young children, particularly those issues that may represent barriers. Three major themes emerged from the study: 1) perceived prevalence, pattern and impact of early childhood caries, 2) perceived causes of decay in childhood, and 3) potential solutions.

Dental caries was clearly identified as a problem by all participants. Most reported that there had been a reduction in dental disease across Australia but certain distinct sub-groups within rural populations appeared to suffer disproportionately. The perceived impact on users included pain and discomfort, future dental problems, orthodontic problems, speech problems, lowered self-esteem for the child and guilt or shame for the mother.

There were two main causes of decay in young children reported by all participants, diet and feeding practices and dental services attendance. All groups were aware of the importance of sugars in the development of caries. Failure on the part of the parent to seek dental care early enough for their child was the second most common explanation put forward by all participants in the study.

The paper goes on to describe potential solutions to the main barriers faced by very young children living in rural areas. The majority of the participants agreed that oral health should be part of routine anticipatory guidance provided for infants and that in order to be effective, activities needed to begin well before most children first see a dentist. Interestingly, despite the feeling that early interventions and anticipatory guidance was critical to preventing caries, the dentists themselves preferred not to see the children until they were at least two years of age and often older.

This paper gives an interesting view into the attitudes and perceptions of health care professions in a one district of Australia. It would be valuable to extend the current study both nationally and internationally to examine the views of health care professionals working in socially deprived areas, to see if similar views are held across the professions.

Dr K. B. Hill, Lecturer in Behavioural Science and Dental Public Health, University of Birmingham 